\title{
El gasto de los cruceristas en Uruguay 2008-2010
}

\section{Wiston Adrián Risso}

Universidad de la República Oriental del Uruguay (Uruguay)

\begin{abstract}
Resumen: En los últimos años el turismo de cruceros se ha expandido en Uruguay. En 2009/10 arribaron 292.048 cruceristas generando un gasto promedio de U\$S 61,05. El presente estudio utiliza las encuestas de cruceristas de 2008/09 y 2009/10 para analizar el perfil de visitante que determina este nivel de gasto. Se encuentra que el turista argentino reduce el gasto en $29 \%$ y $41 \%$ el gasto por persona mientras que el brasileño lo aumenta en $21 \%$ y $16 \%$ por persona. La probabilidad de gastar es de $86 \%$ y $85 \%$ en cada periodo, el desagrado por los precios reduce esta probabilidad en 7 y 4 puntos porcentuales.
\end{abstract}

Palabras clave: Cruceros; Uruguay; Gasto; Precios; Heckman; Modelo Probit.

\section{Title: The Cruise Passengers' Expenditure in Uruguay 2008-2010}

Abstract: In the last years the cruise tourism has increased in Uruguay. In 2009/10 arrived 292,048 cruise passengers producing an average expenditure of U\$S 61.05. The present study uses the cruise surveys of 2008/09 and 2009/09 to analyze the cruise passenger profile determining this expense level. We found that the Argentinean tourist reduces the expenditure in $29 \%$ and $41 \%$, whereas the Brazilian tourist increases the average expenditure in $21 \%$ and $16 \%$ per head. The probability of spending is $86 \%$ and $86 \%$ in each period, and the displeasure for the prices decreases the probability in 7 and 4 perceptual points

Keywords: Cruisers; Uruguay; Expenditure; Prices; Heckman; Probit Model.

\footnotetext{
i Wiston Adrián Risso es doctor en economía $(\mathrm{PhD})$ por la Universidad de Siena (Italia). Es investigador del Instituto de Economía (IECON) y del Instituto de Estadística (IESTA) de la Universidad de la República Oriental del Uruguay (UdelaR). e-mail: arisso@ iecon.ccee.edu.uy

El autor desea agradecer al director de estadística del Mintur, Victor Sosa y a la responsable de cruceros del Mintur, Ana María Pascale por permitirme presentar el trabajo en el Ministerio de Turismo y Deporte de Uruguay (Mintur) y por sus útiles comentarios. Asimismo deseo agradecer el apoyo del Instituto de Economía y del Instituto de Estadística de la Universidad de la República Oriental del Uruguay.
} 


\section{Introducción}

El mercado mundial de cruceros ha presentado un constante crecimiento en las últimas décadas. Desde los años 80 viene aumentando su demanda sostenidamente renovando su enfoque al modificar el concepto de lo que era hacer cruceros y hacer vacaciones en alta mar. Aunque, de acuerdo a la Asociación de Cruceros de Florida y el Caribe (FCCA) la región del Caribe sigue siendo el destino preferido por los turistas, contando con $37,02 \%$ de todos los itinerarios en 2009, ya existen cruceros en prácticamente todos los destinos marítimos del mundo. Por otra parte, se ha percibido que el $80 \%$ de los cruceristas piensa que viajar en crucero es una manera importante para estudiar destinos a los cuales se podría regresar posteriormente como turista, alojándose y permaneciendo uno o varios días en la ciudad.

Según Losano y Torrejón (2006) al contrario de lo que en principio pudiera parecer, no es un tipo de vacaciones privativo de la tercera edad, ni de las clases económicas más pudientes como lo demuestra la edad media del crucerista y los ingresos por unidad familiar (aproximadamente 50.000 dólares para el caso de Estados Unidos). Esto es debido a la oferta de itinerarios cada vez más cortos, por lo tanto, menos costoso y más adaptado a los gustos y exigencias de segmentos específicos de población.

Desde hace algunos años América del Sur ha comenzado a recibir, durante prácticamente todo el año pero más intensamente en la temporada de verano, una cantidad cada vez mayor de barcos. Los circuitos preferidos dentro de la región comprenden, entre otras, las ciudades Río de Janeiro, Punta del Este, Buenos Aires, Montevideo, Ushuaia, Puerto Montt y Valparaíso. En algunos de estos puertos se procede al recambio de pasajeros y de tripulación.

El objetivo del presente estudio es determinar de qué depende el gasto de los cruceristas que llegan a Uruguay. Identificar los factores que favorecen o van en contra de un mayor gasto, es importante a la hora de plantear una estrategia de desarrollo local, aplicando políticas que favorezcan aquellos factores que promueven un mayor gasto y desmotivando aquellos que lo desfavorecen.

El presente trabajo se organiza de la siguiente manera. En la sección 2 se hace una revisión de la literatura sobre el gasto de los cruceristas y se realiza una descripción reciente de la situación de los cruceros en Uruguay. La sección 3 describe los datos utilizados y expone la metodología aplicada. En la sección 4 se analizan los resultados para el gasto en sus diferentes categorías (Gasto total, en compras, en alimentación, en tours y en transporte). En la sección 5 se analizan los determinantes de que el turista declare desagrado por los precios. Por último, en la sección 6 se delinean las principales conclusiones del estudio.

\section{Antecedentes y situación de los cruceros en Uruguay}

Hay pocos estudios realizados sobre el gasto de los cruceristas, tal vez uno de los primeros trabajos sobre este tipo de turismo es el de Mescon y Vozikis (1985), en el que usando un modelo input-output estudian la importancia de los efectos directos, indirectos e inducidos de los cruceros sobre la región de influencia del puerto de Miami.

Dwyer y Forsyth (1998) estudian el turismo de cruceros en Australia concluyendo que las principales fuentes de beneficios para el país provienen de los impuestos cobrados sobre el gasto de los cruceros y de los efectos del tipo de cambio. Por su parte, un estudio de (Wood, 2000) analizando los cruceros en el Caribe sugiere que es posible que los beneficios netos que sobre las economías locales deje el turismo de cruceros en esa región sean ínfimos o negativos dada la poca participación de agentes locales y la limitada regulación e imposición fiscal de la actividad. (Besteiro, 2004), (Esteban, 2000), (Ferradás, 2001, 2002) hablan de la importancia del turismo náutico, en el que el turismo de cruceros está inmerso, como estrategia de diversificación de la actividad económica de las comunidades receptoras.

Chase y Alon, 2002) analizan el impacto del turismo de cruceros en la economía de Barbados, pero utilizando datos del gasto del turista en lugar del gasto del crucerista. En este se concluye que la influencia del crucerismo en Barbados no es significativa, contrario a lo que sucede con el turismo de estancia.

McCarth, (2003) menciona el aparente dilema que enfrentan las localidades receptoras de cruceros entre los impactos económicos positivos y los impactos ambientales negativos del turismo de cruceros. El autor concluye que es indispensable producir estudios que, con un enfoque más riguroso de lo normal, permitan hacer una mejor medición de los costos y los beneficios asociados.

Seidl y otros $(2006,2007)$ cuestionan seriamente la conveniencia de impulsar el turismo de cruceros como estrategia de desarrollo local y regional en Costa Rica.

Cuellar-Río y Kido-Cruz (2008) usando una encuesta de 365 cruceristas en el puerto de Santa Cruz durante la temporada 2005-2006 analizan el perfil y gasto de los visitantes en Bahías de Huatulco (México). Ellos concluyen que el nivel de satisfacción y la decisión de compra de tour en Huatulco producen diferencias estadísticamente significativas en el gasto medio de los cruceristas. La reducción del gasto de un turista bajamente satisfecho respecto del altamente satisfecho va de U\$S 30 a US\$ 42 .

Brida y otros (2010) analizan el perfil de turistas que está relacionado a los diferentes gastos hechos por los visitantes que llegan en cruceros a Cartagena de Indias. El perfil que encuentra es de cruceristas que gastan más en joyas y en general, gastan más en todas las categorías, son más jóvenes tienen ingresos altos. Los grandes consumidores en joyas son mujeres residentes en Estados Unidos. Están viajando por primera o segunda vez en un crucero, permaneciendo un período más extenso en Cartagena de Indias y tienden a viajar en grupos.

Brida y Risso (2010) estudian el gasto y la probabilidad de regresar de los cruceristas en Costa Rica. Se encuentra que el gasto depende positivamente de los visitantes con un ingreso superior a los U\$S 76.000, el número de 
horas fuera del crucero y está afectado negativamente por el hecho de ser soltero, respecto a otros estados civiles. Los norteamericanos que representan más del $60 \%$ de los pasajeros no tienen impacto en el gasto. Por otra parte, la probabilidad de volver a Costa Rica depende positivamente del grado de satisfacción declarado y de las horas fuera de la nave.

\section{Descripción de la situación reciente de Cruceros en Uruguay}

De acuerdo al Anuario Estadístico 2010 del Ministerio de Turismo y Deporte del Uruguay (MinTur) en la temporada de cruceros 2009/10 que va desde noviembre de 2009 a abril 2010, la cantidad de cruceros arribados ascendió a 179, de los cuales 96 corresponden a Punta del Este y 83 a Montevideo. Como se observa en la figura 1 el arribo de cruceros ha presentado un continuo crecimiento desde 2004/05 hasta 2007/08, en 2008/09 se observó una caída del -0,6\%. Esta caída fue debido a un descenso en los arribos de cruceros a Montevideo, ya que el crecimiento en Punta del Este fue continuo en todo el periodo. Finalmente en 2009/10 se observó un incremento total de 8,5\%, con un $12 \%$ de arribos a Punta del Este y un 5\% a Montevideo.

Es interesante destacar aquí, que si bien hasta la temporada 2007/08 la mayoría de los cruceros arribaba a Montevideo, a partir del 2008/09 se revierte la situación pasando Punta del Este a tener el mayor número de arribos.

La cantidad de personas que llegaron a las costas de Uruguay durante la temporada 2009/10 fue 356.198, de las cuales un total de 218.024 personas arribaron a Punta del Este y 138.174 a Montevideo. La proporción de personas que efectivamente desembarcaron estuvo entorno al $82 \%$ en ambos puertos.

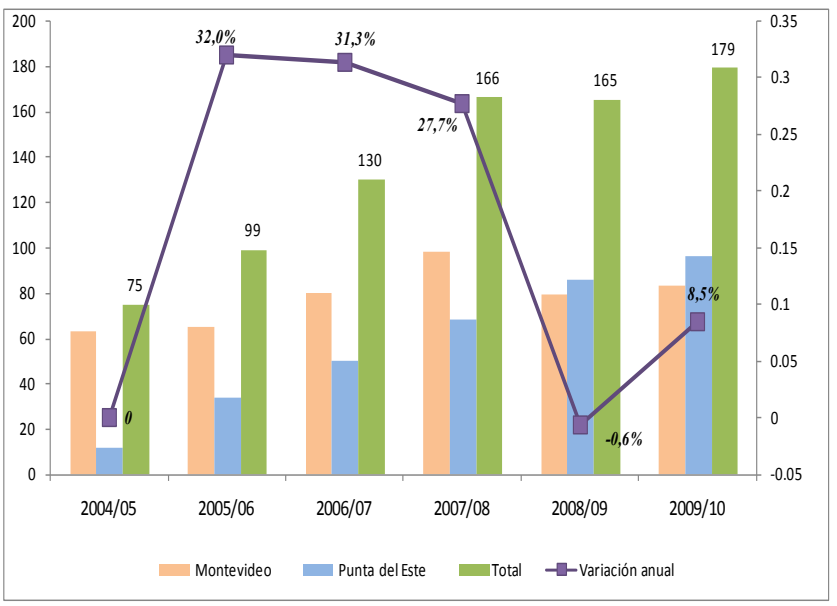

Figura 1. Cruceros Arribados a Uruguay (Montevideo, Punta del Este y Total) y Variación Anual. Fuente: Cálculos propios en base a datos del Ministerio de Turismo y Deportes.
En la figura 2 se puede observar que desde la temporada 2004/05 el número de visitantes que desembarcaron en Uruguay ha crecido con la excepción de la caída en la mencionada temporada de 2008/09. En este caso la temporada 2007/08 marca también un quiebre porque Punta del Este pasa al primer puesto en llegadas de cruceristas mientras que en Montevideo el número tiende a estacionarse.

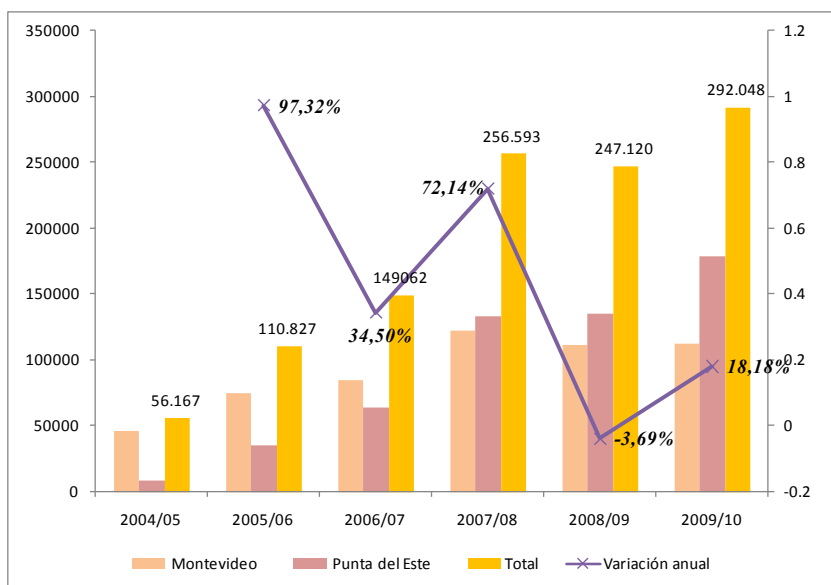

Figura 2. Cruceristas desembarcados en Uruguay (Montevideo y Punta del Este) variación anual. Fuente: Cálculos propios en base a datos del Ministerio de Turismo y Deporte.

Es de notar que si bien el número de visitantes ha crecido desde 2004/05 las tasas de crecimiento se han reducido pasando de $97,32 \%$ en 2005/06 (el máximo incremento) a $18,18 \%$ en 2009/10. Esta desaceleración se ha producido en los dos destinos, pero con más fuerza en Montevideo, en donde pasa de una tasa anual de $60,82 \%$ en $2005 / 06$ a un $0,57 \%$ en $2009 / 10$.

Punta del Este pasa de $283,5 \%$ en $2005 / 06$ a $32,81 \%$ en 2009/10.

Por otro lado, la figura 3 muestra la evolución de los visitantes desembarcados en Montevideo, Punta del Este y el puerto de Buenos Aires en Argentina.

Se puede apreciar que Punta del Este parte de un nivel por debajo de Montevideo y Buenos Aires en 2004/2005 pero en 2007/2008 supera ampliamente a los restantes destinos. Por otra parte es de destacar que el número de cruceristas que desembarcan en Buenos Aires es menor que el número que desembarca en Montevideo durante todo el periodo.

En la tabla 1 observamos que la mayoría de estos turistas son mujeres (59,03\%), en cuanto a la edad la mayoría se encuentra entre los 30 y 64 años.

Como se puede apreciar en la tabla 2, de los cruceristas ingresados en 2009/10 la gran mayoría son brasileños $(52,8 \%)$, en segundo lugar son argentinos $(23,4 \%)$ y los norteamericanos $(12,2 \%)$ ocupan el tercer lugar.

En la temporada 2009/10 los turistas gastaron U\$S 17.830.909 de los cuales el 61,5\% provino de Brasileños, 


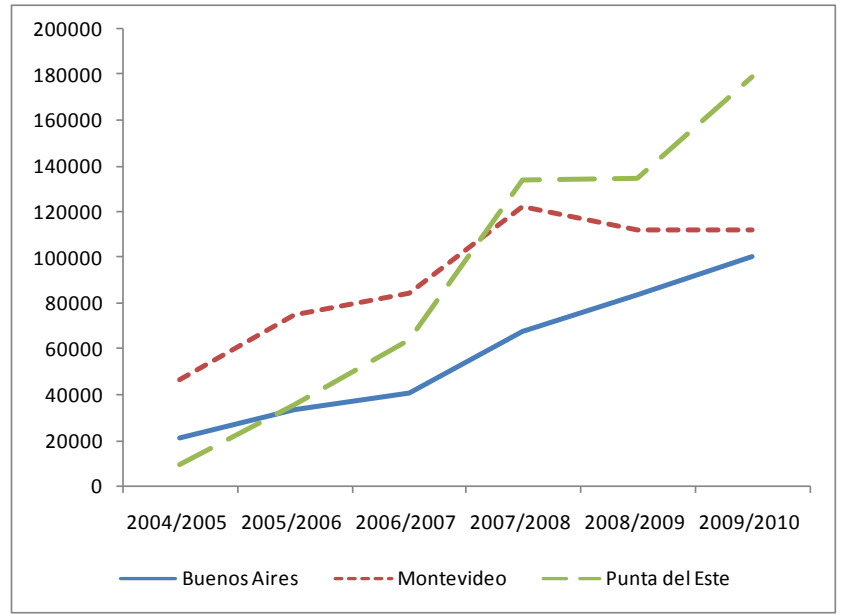

Figura 3. Evolución de los cruceristas desembarcados en Montevideo, Punta del Este y Buenos Aires. Fuente: Cálculos Propios en base a datos del Ministerio de Turismo y Deportes y de la Administración General de Puertos de Buenos Aires.

\begin{tabular}{|l|l|l|l|l|}
\hline & Hombres & Mujeres & Totales Edad & \% Edad \\
\hline \hline Menos de 18 años & 10.377 & 10.174 & 20.551 & $7,04 \%$ \\
De 18 a 29 años & 13.127 & 22.248 & 35.375 & $12,11 \%$ \\
De 30 a 64 años & 74.754 & 103.757 & 178.511 & $61,12 \%$ \\
De 65 y más años & 21.385 & 36.226 & 57.611 & $19,73 \%$ \\
Totales por Sexo & 119.643 & 172.405 & 292.048 & $100 \%$ \\
\% por Sexo & $40,97 \%$ & $59,03 \%$ & $100 \%$ & \\
\hline
\end{tabular}

Tabla 1. Edad y Sexo de los Cruceristas. Fuente: en base a los datos del Ministerio de Turismo y Deporte.

\begin{tabular}{|l|l|l|}
\hline Nacionalidad & Total & Porcentaje \\
\hline \hline Brasil & 154.061 & $52,8 \%$ \\
Argentina & 68.328 & $23,4 \%$ \\
Norteamérica & 35.546 & $12,2 \%$ \\
Europa & 15.65 & $5,4 \%$ \\
Otros América & 12.838 & $4,4 \%$ \\
Uruguay & 356 & $0,1 \%$ \\
Otros/Sin dato & 5.269 & $1,8 \%$ \\
\hline \hline Total & $\mathbf{2 9 2 . 0 4 8}$ & $\mathbf{1 0 0 , 0 \%}$ \\
\hline
\end{tabular}

Tabla 2. Cruceristas desembarcados en Uruguay, según nacionalidad en la temporada 2009/10 Fuente: en base a los datos del Ministerio de Turismo y Deporte

\begin{tabular}{|l|l|l|}
\hline Nacionalidad & Total Gasto & Porcentaje \\
\hline \hline Brasil & 10.961 .578 & $61,5 \%$ \\
Argentina & 2.444 .492 & $13,7 \%$ \\
Norteamérica & 2.125 .379 & $11,9 \%$ \\
Europa & 911.632 & $5,1 \%$ \\
Otros América & 906.791 & $5,1 \%$ \\
Chile & 207.651 & $1,2 \%$ \\
Uruguay & 3.085 & $0,02 \%$ \\
Otros & 270.301 & $1,5 \%$ \\
\hline Total & $\mathbf{1 7 . 8 3 0 . 9 0 9}$ & $\mathbf{1 0 0 , 0 \%}$ \\
\hline
\end{tabular}

Tabla 3. Gasto en USS de los cruceristas en 2009/10 por nacionalidad. Fuente: en base a los datos del Ministerio de Turismo y Deporte.

el 13,7\% de Argentinos, el 11,9\% de Norteamericanos y el resto del mundo participó en un 12,8\%. Es de destacar la importancia que tienen los cruceristas en el total del gasto efectuado si bien fueron la mayoría de los que arribaron a Uruguay con $52,8 \%$ del total de arribos, su participación en el gasto es superior, es de $61,5 \%$. Los argentinos por su parte fueron un $23,4 \%$ pero su participación en el gasto es de $11,9 \%$ (ver figura 4 ).

La figura 4 muestra claramente que los Uruguayos y los Argentinos son los turistas que gastan menos por persona con U\$S 8,67 y U\$S 35,78. Sin embargo, los

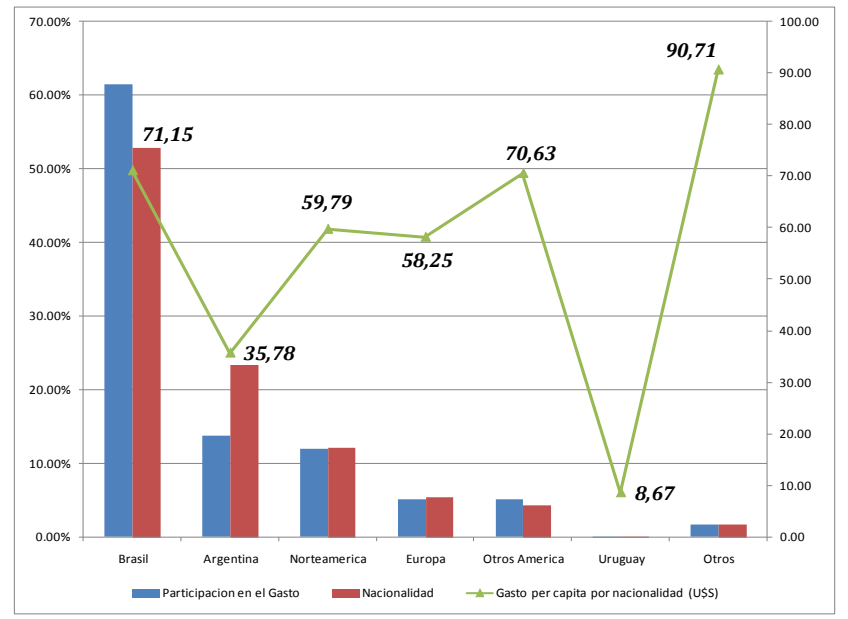

Figura 4. Relación Nacionalidad y Participación en el Gasto de los Cruceristas. Fuente: en base a los datos del Ministerio de Turismo y Deporte

brasileños y el resto del mundo (excluido EEUU, Europa y América) tienen el gasto por persona más alto de U\$S 71,15 y U $\$$ S 90,71 respectivamente, luego sigue el resto de los americanos que gastan U\$S 70,63 una cifra cercana a la de los brasileños. Esto podría considerarse como un primer indicador señalando la importancia que tiene en 
el gasto la llegada de visitantes del resto del mundo, de brasileños y del resto de América.

Es importante aclarar, que el gasto de los nacionales uruguayos es bajo dado que el total de los encuestados de esta nacionalidad residen en el país en la primera muestra, mientras que en la segunda muestra el 31\% reside en Argentina y el resto en Uruguay.

Por otro lado, la Administración General de Puertos de Buenos Aires asegura que el promedio que gasta un crucerista es de U\$S 150 y U\$S 200 por día, lo que estaría por encima de los resultados en Uruguay.

La tabla 4 muestra que se gastaron U $\$$ S 12.655 .804 en Compras (71\% del total) y en segundo lugar U\$S 2.914.781 en alimentación (16,3\%), el resto no llega al 10\%.

La figura 6 nos muestra el gasto ha ido creciendo en el periodo 2005/06 a 2009/10, a excepción de la temporada 2008/09 que como se había mencionado también había presentado un descenso en las personas que arribaron.

\begin{tabular}{|l|l|l|}
\hline Rubro & Gasto en U\$S & Porcentaje \\
\hline Compras & 12.655 .804 & $71,0 \%$ \\
Alimentación & 2.914 .781 & $16,3 \%$ \\
Tour & 1.090 .573 & $6,1 \%$ \\
Transporte & 237.398 & $1,3 \%$ \\
Otro gasto & 932.353 & $5,2 \%$ \\
\hline Total & $\mathbf{1 7 . 8 3 0 . 9 0 9}$ & $\mathbf{1 0 0 , 0 \%}$ \\
\hline
\end{tabular}

Tabla 4. Rubro en el que los cruceristas gastaron en 2009/10Fuente: en base a los datos del Ministerio de Turismo y Deporte.

Nótese de nuevo aquí el cambio en la tendencia en el 2007/08 donde Punta del Este pasa a tener el mayor gasto. Las variaciones anuales indican que a lo largo de todo el periodo las tasas de crecimiento en el gasto han desacelerado hasta caer en 2008/09 para luego presentar un repunte en 2009/10, con un mayor crecimiento en Punta del Este.

En cuanto a los motivos de agrado, en las primeras cinco posiciones aparecen: a) la gente, la cordialidad, la atención; b) la playa y las ramblas; c) la arquitectura, edificios y monumentos, estos tres en ambos puertos. Además en Montevideo aparece el Mercado del Puerto y en Punta del Este la higiene y la seguridad y tranquilidad.

En el total para Uruguay se destaca que los visitantes han valorado con agrado todo $(23,80 \%)$ la gente la cordialidad y la atención (19,91\%), las playas, ramblas y costas $(13,36 \%)$, la arquitectura, edificios y monumentos $(11,36 \%)$, la higiene $(5,7 \%)$ y el mercado del puerto $(3,95 \%)$. Por otro lado, señalan con desagrado nada (67,35\%), los precios $(8,3 \%)$, la higiene $(3,06 \%)$, falta de comercios $(1,88 \%)$ y la mala atención en los servicios $(1,85 \%)$.

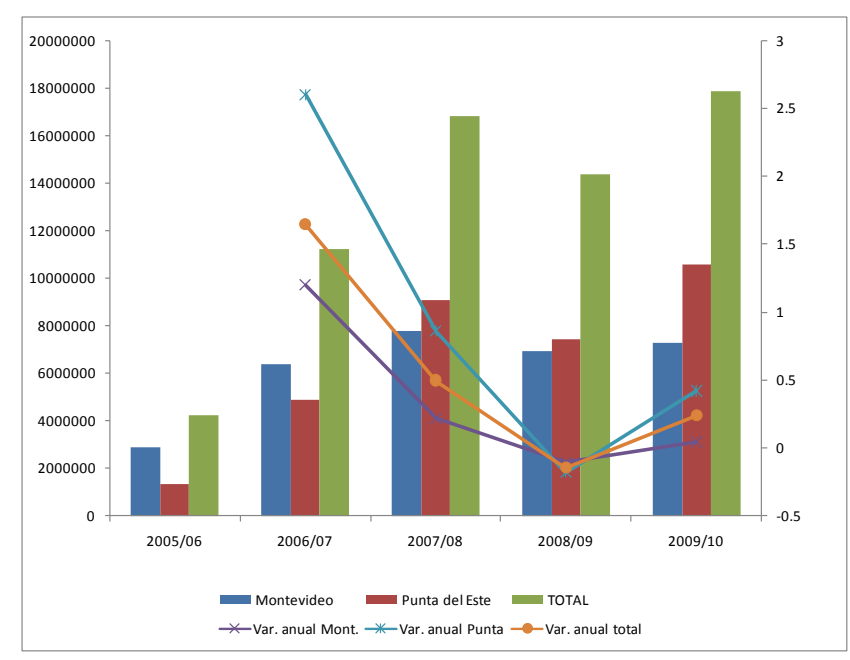

Figura 6. Gasto de los cruceristas total y según puerto y crecimiento anual. Fuente: en base a los datos del Ministerio de Turismo y Deporte.

\section{Datos y Metodología aplicada}

Se utilizaron los microdatos de las Encuestas de Cruceros de 2008/2009 y 2009/2010 realizadas por el departamento de estadísticas del Ministerio de Turismo y Deporte de Uruguay. El marco muestral fue elaborado tomando en cuenta los siguientes puntos: 1) Los cruceros que se quedan 2 días en Montevideo o Punta del Este, se los entrevista el segundo día de su estadía; 2) Los cruceros que están un día en cada puerto, son considerados como barcos distintos en cada visita, para realizar las entrevistas; 3) Se realizan dos muestras independientes con los cruceros que llegan al puerto de Montevideo y con los que llegan a Punta del Este; 4) En cada uno de los puertos se toma el 1\% de la capacidad total de los cruceros que llegan, y la suma de dichas capacidades es la cantidad de cruceros a encuestar en la temporada.

El trabajo se centra en determinar las variables que explican los diferentes tipos de gastos a los efectos de identificar aquellos factores que han favorecido un aumento del gasto como aquellos que lo desfavorecen.

Cuando se estudia el gasto en muestras de corte transversal se recomienda aplicar un modelo econométrico de variable dependiente censurada Tobit (ver Tobin, 1958). Como es sabido variables como el gasto no toman valores negativos y por tanto estimar una regresión lineal no proporcionaría buenos resultados. Sin embargo el modelo Tobit produce estimaciones inconsistentes cuando los datos violan el supuesto de normalidad. En el presente caso sucede esto y por tanto se decidió como modelo principal un modelo de selección de Heckman (ver Cameron y Trivedi, 2009), este modelo tiene la ventaja de que las estimaciones si no son normales ya no serán inconsistentes. Otra ventaja de este modelo es que podremos analizar no solo de qué depende el gasto de estos 
turistas, sino que también de qué depende la decisión de gastar a través de la probabilidad de generar gasto o no.

Song y Li (2008) muestran que la mayoría de los estudios empíricos usan series de tiempo con el objetivo de estudiar la dinámica de la demanda de turismo. Son pocos los trabajos que usan datos de corte transversal de una muestra de turistas (ver Aguiló y Juaneda, 2000). Los datos de corte transversal son muchas veces utilizados en estudios en los cuales se analiza la segmentación del mercado tratando de identificar grupos homogéneos dentro de una población heterogénea. En el presente estudio utilizamos este modelo a los efectos de cuantificar y analizar las características de los cruceristas que afectan los principales tipos de gasto, como se observa en la tabla 4, estos son el gasto total, el gasto en compras y el gasto en alimentación.

En el presente estudio se usa además, un modelo de regresión Probit (ver Long, 1997) a los efectos de analizar cuáles son las variables dentro de la muestra que determinan que se tenga una visión negativa sobre los precios en Uruguay.

Las variables utilizadas son de diferente tipo, algunas continuas, otras discretas y binarias. Para representar el Gasto se utilizaron las variables Gasto Total, Gasto en Compras y el Gasto en Alimentación. Se aplicaron las variables de nacionalidad Brasil, Argentina y EE.UU. dejando como control al resto del mundo. Variables binarias sobre el agrado, como (Gusto) G. Todo, G. Gente, G. Playa, G. Edificios, G. Higiene y G. Mer. Puerto y variables del desagrado, como (Desagrado) D. Nada, D. Precios, D. Higiene, D. Pobreza. Variables de arribos como primera vez (1ra Vez) y el número de visitas anteriores (no. Visitas). Variables sobre la profesión, Profesional (Profesional, técnico, profesor, artista, periodista), Jubilado (Jubilado o Pensionista), Patrón (Patrón, comerciante, industrial, productor agropecuario, empresario) y Empleado (Empleado adm., cajero, vendedor, bancario, empleado público) dejando como control a las otras profesiones. La variable Hombre se tomó como variables de género, tomando 1 cuando el encuestado es hombre y 0 si es mujer. Por último, se consideraron los grupos por edad de 65 años o mayores (ed65), entre 36 y 64 años (ed36a64) dejando como control el grupo de menores de 35 . Se debe hacer la salvedad que para la muestra del 2008/09 la categoría cambiaba al grupo de 30 a 64 años y menores de 30 años.

Hubiera sido interesante contar en la encuesta con variables como el nivel de ingreso, el nivel educativo, el estado civil y si se piensa regresar de nuevo al país. Muchas de estas han resultado significativas en otros estudios.

Las tabla 5 muestra algunas características de las variables usadas en las encuestas tanto para 2008/09 que para 2009/10.

Se observa que en la encuesta también la mayoría de los encuestados son mujeres con una participación que va del $62,9 \%$ al $63,3 \%$. Asimismo, el grupo mayoritario se encuentra entre los 30 y 64 años. Aunque los

\begin{tabular}{|c|c|c|}
\hline & $2008 / 09$ & $2009 / 10$ \\
\hline & $\begin{array}{l}\text { Cruceristas } \\
(\%)\end{array}$ & $\begin{array}{l}\text { Cruceristas } \\
(\%)\end{array}$ \\
\hline \multicolumn{3}{|l|}{ Nacionalidad (\%): } \\
\hline Brasil & $35,1 \%$ & $46,5 \%$ \\
\hline Argentina & $17,8 \%$ & $25,9 \%$ \\
\hline EEUU & $18,4 \%$ & $9,7 \%$ \\
\hline Primera Vez (\%) & $72,3 \%$ & $68,6 \%$ \\
\hline $\begin{array}{ll}\text { Visitas } & \text { Anteriores } \\
\text { (Promedio) } & \end{array}$ & 0,7 & 0,8 \\
\hline \multicolumn{3}{|l|}{ Edad (\% en categoría): } \\
\hline$>64$ & $20,6 \%$ & $21,4 \%$ \\
\hline 30-64 (a) & $65,1 \%$ & $70,4 \%$ \\
\hline$<30$ (a) & $14,4 \%$ & $8,2 \%$ \\
\hline Hombre (\%): & $37,1 \%$ & $36,7 \%$ \\
\hline $\begin{array}{l}\text { Gasto Total por Encuestado } \\
\text { en USS }\end{array}$ & 135,69 & 133,07 \\
\hline Gasto Total por persona USS & 62,06 & 62,72 \\
\hline \multicolumn{3}{|l|}{ Principales Profesiones: } \\
\hline Profesional, Artista (\%) & $32,5 \%$ & $34,2 \%$ \\
\hline Jubilado, pension. (\%) & $31,3 \%$ & $25,4 \%$ \\
\hline Patrón, Comerciant. (\%) & $11,2 \%$ & $14,6 \%$ \\
\hline Empleado, Bancario (\%) & $8,2 \%$ & $7,8 \%$ \\
\hline Total Encuestados & 1803 & 3348 \\
\hline \multicolumn{3}{|c|}{$\begin{array}{l}\text { (a) En } 2008 / 09 \text { las franjas son menores de } 35 \text {, entre } \\
35 \text { y } 64 \text { y mayores de } 64\end{array}$} \\
\hline
\end{tabular}

Tabla 5: algunas características de los datos de las encuestas de cruceros 2008/09 2009/10. Fuente: Encuesta de Cruceros, Ministerio de Turismo y Deporte.

resultados sobre este grupo deben ser relativizados al tratarse del tramo mayor de edades.

Como se mencionó anteriormente, la mayoría de los visitantes son brasileños, argentinos y norteamericanos, lo que se observa también en las encuestas. En los dos periodos cerca del $70 \%$ de los encuestados arriban por primera vez a Uruguay. Se observa que la mayoría de los encuestados entran dentro de las categorías de profesionales, artistas, profesores o jubilados y pensionistas. 


\section{Resultados Empíricos}

Las tablas $6,7,8$ y 9 muestran los resultados para el gasto total, en alimentación y en compras para los dos periodos 2008/09 y 2009/10. En la tabla 10 se puede apreciar el gasto promedio y la probabilidad de gasto previsto por los modelos en ambos periodos. Por último, la tabla 11 muestra los resultados de los determinantes del desagrado por los precios.

\section{Determinantes Gasto total}

En las tablas 6 y 8 se observa los resultados para el gasto usando el modelo Tobit y el de selección de Heckman. En todos los casos el de Heckman produce los mejores resultados. El mínimo AIC se observa con Heckman y la mejor predicción en relación al promedio de la encuesta se produce con este modelo. Mientras la media fue de U\$S 52,81 y U\$S 61,05, el modelo de Tobit predice U\$S 27,11 y U\$S 18,20 esto es efecto de la inconsistencia de las estimaciones cuando los datos no son normales. En cambio, para el modelo de Heckman tenemos U\$S 62,54 y U\$S 62,12 que son resultados mucho más cercanos a la realidad.

La nacionalidad del crucerista es una variable muy significativa en el gasto total. Mientras que el crucerista brasileño determina un incremento del $21 \%$ y $16 \%$ del gasto medio, el crucerista argentino tiene el efecto contrario con una caída de $-29 \%$ y $-41 \%$ del gasto total medio. Por otra parte el crucerista norteamericano tuvo un impacto negativo significativo en el periodo 2008/09 con una caída $-17 \%$ del gasto total medio. Se observa que en 2008/09 la probabilidad de gastar es de $86 \%$ y $85 \%$ en cada periodo. El ser brasileño determina un incremento en la probabilidad de comprar de 5 puntos porcentuales en el primer periodo, pero el mismo tipo de crucerista reduce la probabilidad en 2 puntos en 2009/10. En cambio el visitante argentino determina una caída en la probabilidad de comprar de 15 y 10 puntos porcentuales.

El efecto de la variable agrado y desagrado es diferente en los dos periodos. Se puede identificar que aquellos que declaran gusto por los monumentos y edificios han reducido el gasto medio en $23 \%$ y $5 \%$ respectivamente. La variable agrado por Todo y Playas ha disminuido la probabilidad de compra en 7 y 3 puntos porcentuales para el primero y en 10 y 4 puntos para el segundo. Una variable interesante es el desagrado por los precios, nótese que declarar desagrado por los precios reduce la probabilidad de compra en 7 y 4 puntos porcentuales en cada periodo. Sin embargo, mientras en el primer periodo quien decidió comprar pese a declarar desagrado aumenta el gasto en $19 \%$, ya en el segundo periodo reduce el gasto en $17 \%$.

El hecho de que sea la primera vez o que se haya visitado varias veces el Uruguay no tiene importancia en el gasto total, como se puede apreciar ninguna de estas variables es significativa.

En cuanto a las profesiones es de destacar que quien es patrón, comerciante, industrial, productor agropecuario, empresario tiene el impacto más positivo y significativo en ambos periodos. Estos aumentan el gasto en $34 \%$ y $47 \%$ sobre la media, mientras que aumentan la probabilidad de comprar en 8 y 5 puntos porcentuales.

En cuanto al género del crucerista, el declarar ser hombre pareciera tener un impacto del $2 \%$ sobre el gasto en ambos periodos pero no es significativo. Sin embargo sí es significativo el hecho que aumenta la probabilidad de gastar en 4 y 2 puntos porcentuales en cada periodo.

Por último, el grupo etario de más de 65 años aumenta el gasto y es significativo en ambos periodos respecto a los otros grupos etarios aumentando el gasto medio en $22 \%$ en ambos periodos.

De esto se puede concluir que el perfil del crucerista que ha impactado con un mayor gasto es el del crucerista brasileño patrón, comerciante, industrial, productor agropecuario, empresario con más de 65 años. Mientras que el perfil más negativo es el de un crucerista argentino menor de 65 años que ha manifestado desagrado por los precios.

\section{Determinantes del Gasto en compras}

En cuanto al gasto en compras se observa un comportamiento similar en cuanto a las nacionalidades respecto al observado en el gasto total. Los brasileños tienen un impacto positivo en el gasto en compra respecto al resto del mundo, mientras que los argentinos tienen el efecto contrario. Mientras que en el primer periodo es significativa la reducción en el gasto por parte de los argentinos en $43 \%$ y el aumento del gasto por parte de los brasileños en $15 \%$, en el segundo periodo se mantienen los signos, pero lo que es significativo es la decisión de comprar, la cual aumenta en el caso de los brasileños en 2 puntos porcentuales y disminuye en 9 puntos en el caso de los argentinos.

En cuanto a las cosas que agradaron o que desagradaron, no se encuentran resultados claros, en el primer periodo el gusto por todo fue significativo, pero en el segundo periodo el gusto por la higiene, las playas y el mercado del puerto determinan una reducción en la probabilidad de compra de 10 puntos porcentuales. En cuanto a las cosas que desagradan, los precios reducen significativamente la probabilidad de compra en el primer periodo en 7 puntos porcentuales. Por otro lado, la pobreza también ha reducido significativamente la probabilidad de comprar en 8 y 14 puntos porcentuales. Nótese que en el primer periodo la probabilidad de compra es de $30 \%$ y en el segundo de $32 \%$.

De nuevo aquí, el hecho de ser la primera vez o haber visitado antes Uruguay no tiene importancia en el gasto en compras.

En cuanto a las profesiones los resultados son ambiguos. En el primer periodo, los jubilados tienen un impacto negativo en las compras bajando tanto el gasto como la probabilidad de comprar. Pero no 
es significativo en el segundo periodo. El ser patrón tiene un impacto positivo diferente en ambos periodos, mientras que en el primero aumenta la probabilidad de comprar en 12 puntos, en el segundo lo que aumenta es el gasto en $46 \%$.

En cuanto al género, el ser hombre solo presenta una reducción en la probabilidad de compra en el primer periodo.

Por último, la grupo entre 35 y 64 años reduce la probabilidad de compra en 5 puntos solo en el primer periodo, respecto a los demás grupos etarios.

Si tenemos que definir un perfil que favorezca el gasto en compras es el del crucerista brasileño, tal vez mujer que ha manifestado desagrado por la pobreza y que pertenece a la categoría profesional de patrón, comerciante, industrial, productor agropecuario, empresario. Mientras que el turista argentino sigue siendo el que ha impactado más negativamente el gasto en compras.

\section{Determinantes del Gasto en Alimentación}

En cuanto al gasto en alimentación ninguna variable parece afectar el nivel del gasto en 2008/09. El visitante argentino reduce el gasto en $37 \%$ y la probabilidad de gastar en alimentos en 5 puntos en 2009/10 respecto al resto de las nacionalidades.

La variable agrado por monumentos y edificios ha impactado reduciendo la probabilidad de gasto en alimentación en 6 y 5 , pero no es significativa su dependencia con el gasto. El desagrado por los precios baja la probabilidad de gasto en 7 puntos en el primer periodo, pero no es significativo en el segundo periodo.

Una vez más, el hecho de ser la primera vez o haber estado en el pasado en el país no tiene importancia a la hora de explicar el gasto en alimentación.

En cuanto a las profesiones, no son significativas en el primer periodo. Sin embargo estas variables son significativas en el segundo periodo de la muestra. Dentro de estas últimas se destaca la de patrón, empresario con $78 \%$ en el gasto aunque reduciendo la probabilidad de compra en 7 puntos porcentuales.

Son los hombres los que tienen un impacto positivo significativo en la probabilidad de gasto en alimentación con 9 y 5 puntos porcentuales.

Por último, la edad no parece importante a la hora de explicar el gasto en alimentación en 2008/09. El grupo de entre 30 y 64 años aunque disminuyen la probabilidad de gasto en 3 puntos porcentuales, aumentan el gasto en $20 \%$.

Como se puede observar aquí, el perfil que beneficia significativamente es el del crucerista que no sea argentino, tal vez hombre.

\section{Modelo Probit para la probabilidad del desagrado por los precios}

Si bien son pocos los que manifiestan desagrado por los precios, alrededor del $8 \%$ y $9 \%$ de los encuestados en cada periodo. Es interesante ver de qué depende que

\begin{tabular}{|c|c|c|c|c|}
\hline \multirow[b]{2}{*}{ Var. Depend. } & \multicolumn{4}{|c|}{ Gasto en Alimentación } \\
\hline & Tobit ${ }^{(\mathbf{b})}$ & $\operatorname{Heckman}^{(\mathrm{c})}$ & Selección ${ }^{(c)}$ & $\mathrm{dP} / \mathrm{dx}$ \\
\hline Brasil & $-0,60[-1,30]$ & $0.12[0,03]$ & $0.01[0,08]$ & 0.00 \\
\hline Argentina & $-1,71[-2,91]^{* * *}$ & $-4.17[-0,08]$ & $-0.13[-1,38]$ & -0.04 \\
\hline EE.UU. & $-0,26[-0,46]$ & & & \\
\hline G. Todo & $-0,11[-0,25]$ & & & \\
\hline G. Gente & $-0,50[-0,86]$ & & & \\
\hline G. Edificios & $-1,34[-1,81]^{*}$ & $-6,00[-0,07]$ & $-0,21[-1,66]^{*}$ & -0.06 \\
\hline G. Playa & $0.61[0,89]$ & & & \\
\hline G. Higiene & $-1,23[-1,08]$ & & & \\
\hline G. Mer. Puerto & $-0,61[-0,46]$ & & & \\
\hline D. Nada & $-0,22[0,45]$ & & & \\
\hline D. Precios & $-1,43[-1,73]^{*}$ & $-7,11[-0,07]$ & $-0,26[-1,92]^{*}$ & -0.07 \\
\hline D. Higiene & $0,32[0,31]$ & & & \\
\hline D. Pobreza & $-0,57[-0,30]$ & & & \\
\hline 1ra Vez & $-0,58[-0,89]$ & & & \\
\hline No. Visitas & $0,13[0,61]$ & & & \\
\hline Profesional & $-0,31[-0,58]$ & & & \\
\hline Jubilado & $-1,61[-2,37]^{* *}$ & & & \\
\hline Patrón & $-0,39[-0,56]$ & & & \\
\hline Empleado & $-0,31[-0,42]$ & & & \\
\hline Hombre & $1,53[4,11]^{* * *}$ & $8,73[0,07]$ & $\begin{array}{r}0,31 \\
{[4,52]^{* * *}} \\
\end{array}$ & 0.09 \\
\hline ed36a64 (a) & $-0,06[-0,11]$ & & & \\
\hline ed65 & $-1,22[-1,55]$ & & & \\
\hline Constante & $-3,67[-3,75]^{* * *}$ & $-48,74[-0,07]$ & $\begin{array}{r}-0,87[- \\
14,94]^{* * *} \\
\end{array}$ & \\
\hline Obs. no censuradas & 380 & 380 & & \\
\hline Chi2 & (LR) $73,63 * * *$ & $\begin{array}{r}\text { (Wald) } \\
29.24 * * * \\
\end{array}$ & No sesgo & \\
\hline media var. Ind. & 6.13 & 6.13 & & $21 \%(\mathrm{~d})$ \\
\hline Predicción & 0.00 & 0.00 & & \\
\hline $\begin{array}{l}\text { Criterio Akaike } \\
\text { (AIC) }\end{array}$ & 3526.075 & two step & & \\
\hline
\end{tabular}

Tabla 6. Regresión de Tobit para el Gasto de los cruceristas en Uruguay 2008/2009*significación al 10\%, **significación al 5\%, *** significación al 1\%. (a) La encuesta de 2009/10 usa ed30-64. (b) Coeficientes y estadístico $\mathrm{W}$ entre paréntesis [] para el modelo Tobit. (c) Modelo de eckman primera columna coeficientes, segunda columna ecuación selección y la tercera muestra el efecto en la probabilidad de gastar. (d) probabilidad de gastar. Fuente: Cálculos propios en base a las encuestas de cruceros suministradas por el Ministerio de Turismo y Deporte de Uruguay.

alguien declare desagrado por los precios porque podría ser un indicador de reducción en el gasto. Se estimó un modelo Probit a los efectos de estudiar de que depende la probabilidad de que alguien declare que le desagradan los precios, la tabla 11 muestra los resultados.

En ambos casos, el ser argentino o brasileño aumenta la probabilidad de manifestar un desagrado por el precio. En ambos casos el impacto marginal es más alto en el caso de los argentinos que el de los brasileños. Un argentino aumenta la probabilidad en $15 \%$ y $2 \%$ en cada periodo y un brasileño en $13 \%$ y $1 \%$. Por otro lado, los norteamericanos parecen no disgustarse por los precios 


\begin{tabular}{|c|c|c|c|c|c|c|c|c|}
\hline \multirow[b]{2}{*}{ Var. Depend. } & \multicolumn{4}{|l|}{ Gasto Total } & \multicolumn{4}{|c|}{ Gasto en Compras (Shopping) } \\
\hline & Tobit $^{(\mathbf{b})}$ & Heckman $^{(\mathrm{c})}$ & Selección (c) & $d P / d x$ & Tobit $^{(a)}$ & Heckman(c) $^{(\mathrm{s})}$ & Selección (c) & $d P / d x$ \\
\hline Brasil & $0.46[3,41]^{* * *}$ & $0.21[3,29]^{* * *}$ & $0.25[2,46]^{* * *}$ & 0.05 & $0.89[2,11]^{* *}$ & $0,10[0,85]$ & $0,15[2,13]^{* *}$ & 0.05 \\
\hline Argentina & $-1.12[-6,72]^{* * *}$ & $-0.29[-3,11]^{* * *}$ & $\begin{array}{ll}-0,57 & {[-} \\
5,43]^{* * *} & \\
\end{array}$ & -0.15 & $-2.29[-4,07]^{* * *}$ & $-0.24[-1,03]$ & $-0.43[-4,46]^{* * *}$ & -0.14 \\
\hline EE.UU. & $-0.10[-0,62]$ & $-0.17[-2,34]^{* *}$ & $0,05[0,43]$ & 0.01 & $0,44[0,84]$ & & & \\
\hline G. Todo & $-0.43[-3,25]^{* * *}$ & $-0.11[-1,74]^{*}$ & $\begin{array}{ll}-0,30 & {[-} \\
3,25]^{* * *} & \\
\end{array}$ & -0.07 & $1,34[3,25]^{* * *}$ & $0,05[0,31]$ & $0,28[3,85]^{* * *}$ & 0.10 \\
\hline G. Gente & $-0,51[-3,11]^{* * *}$ & $-0.07[-0,86]$ & $\begin{array}{ll}-0.36 & \\
3,22]^{* * *}\end{array}$ & -0.09 & $-0,43[-0,80]$ & & & \\
\hline G. Edificios & $-0.77[-3,82]^{* * *}$ & $-0.23[-2,34]^{* *}$ & $\begin{array}{ll}-0,45 & {[-} \\
3,27]^{* * *} & \\
\end{array}$ & -0.12 & $0,25[0,40]$ & $-0, .35[-2,13]^{* *}$ & $0.12[0,99]$ & 0.04 \\
\hline G. Playa & $-0.72[-3,44]^{* * *}$ & $-0.27[-2,73]^{* * *}$ & $\begin{array}{ll}-0,38 & {[-} \\
2,69 & \end{array}$ & -0.10 & $0,19[0,29]$ & & & \\
\hline G. Higiene & $-0.02[-0,06]$ & & & & $0,14[0,15]$ & $-0,33[-1,30]$ & $0,09[0,49]$ & 0.03 \\
\hline G. Mer. Puerto & $-0.01[-0,02]$ & & & & $-3,51[-2,32]^{* *}$ & & & \\
\hline D. Nada & $0.15[1,01]$ & & & & $-0.22[-0,48]$ & & & \\
\hline D. Precios & $-0.08[-0,37]$ & $0.19[2,03]^{* *}$ & $-0.28[-2,16]^{* *}$ & -0.07 & $-1.22[-1,65]^{*}$ & $0.12[0,63]$ & $-0.21[-1,73]^{*}$ & -0.07 \\
\hline D. Higiene & $0,46[1,55]$ & & & & $-3.95[-3,30]^{* * *}$ & $-0.49[-0,99]$ & $-0,69[-3,24]^{* * *}$ & -0.19 \\
\hline D. Pobreza & $-0.21[-0,42]$ & $0,78[3,02]^{* * *}$ & $-0,54[-1,83]^{*}$ & -0.15 & $-0.89[-0,50]$ & $1,03[1,97]^{* *}$ & $-0,24[-0,73]$ & -0.08 \\
\hline 1ra Vez & $0,05[0,24]$ & & & & $0.70[1,11]$ & & & \\
\hline No. Visitas & $-0.03[-0,41]$ & & & & $0,17[0,82]$ & & & \\
\hline Profesional & $0,54[3,31]^{* * *}$ & $0,22[2,89]^{* * *}$ & $0,30[2,69]^{* * *}$ & 0.06 & $0,83[1,63]^{*}$ & & & \\
\hline Jubilado & $-0,17[-0,88]$ & $-0,16[-1,78]^{*}$ & $0.02[0,12]$ & 0.00 & $-1.62[-2,53]^{* * *}$ & $-0,34[-1,83]^{*}$ & $-0,37[-4,68]^{* * *}$ & -0.12 \\
\hline Patrón & $0,80[3,86]^{* * *}$ & $0,34[3,53]^{* * *}$ & $0.46[2,97]^{* * *}$ & 0.08 & $2.28[3,62]^{* * *}$ & $0.22[1,29]$ & $0,33[3,30]^{* * *}$ & 0.12 \\
\hline Empleado & $-0,03[-0,16]$ & $0,01[0,14]$ & $-0.00[-0,02]$ & 0.00 & $0.61[0,87]$ & & & \\
\hline Hombre & $0,24[2,22]^{* *}$ & $0,02[-0,31]$ & $0,21[2,60]^{* * *}$ & 0.04 & $-0.79[-2,28]^{* *}$ & $0,00[0,03]$ & $-0.15[-2,34]^{* *}$ & -0.05 \\
\hline ed36a64(a) & $0,10[0,62]$ & & & & $-0,79[-1,66]^{*}$ & $0,08[0,73]$ & $-0,15[-2,16]^{* *}$ & -0.05 \\
\hline ed65 & $0,33[1,46]$ & $0,22[2,80]^{* * *}$ & $0,05[0,39]$ & 0.01 & $0,04[0,06]$ & & & \\
\hline Constante & $2,74[9,43]^{* * *}$ & $3,77[40,10]^{* * *}$ & $1,08[9,17]^{* * *}$ & & $-3,29[-3,52]^{* * *}$ & $3,61[6,36]^{* * *}$ & $-0,31[-3,71]^{* * *}$ & \\
\hline Obs. no censuradas & 1522 & 1522 & & & 557 & 557 & & \\
\hline Chi2 & (LR) $205.25 * * *$ & (Wald) $102.67 * * *$ & No Sesgo & & (LR) $136.73^{* * *}$ & (Wald) $27.77^{*}$ & No Sesgo & \\
\hline media var. Ind. & 58.21 & 58.21 & & $0,86^{(\mathrm{d})}$ & 21.26 & 21.26 & & $0,30^{(\mathrm{d})}$ \\
\hline Predicción & 27.11 & 62.54 & & & 0.08 & 22.16 & & \\
\hline Criterio Akaike (AIC) & 7440.081 & 5605.525 & & & 4785.942 & 3758.168 & & \\
\hline
\end{tabular}

Tabla 7. Regresión de Tobit para el Gasto de los cruceristas en Uruguay 2008/2009*significación al 10\%, **significación al 5\%, *** significación al 1\%. (a) La encuesta de 2009/10 usa ed30-64. (b) Coeficientes y estadístico W entre paréntesis [] para el modelo Tobit. (c) Modelo de Heckman primera columna coeficientes, segunda columna ecuación selección y la tercera muestra el efecto en la probabilidad de gastar (d) probabilidad de realizar el gasto. Fuente: Cálculos propios en base a las encuestas de cruceros suministradas por el Ministerio de Turismo y Deporte de Uruguay

aunque esto no es significativo en ninguno de los periodos.

Las variables sobre los gustos no fueron significativas en el primer periodo, siendo positivamente significativo en el segundo periodo el agrado por todo, playas e higiene. El desagrado tampoco es significativo en el primer periodo pero sí lo es en el segundo.

Parecería que a mayor gasto total no tiene un impacto significativo en la probabilidad de declarar desagrado por los precios.

Las profesiones tampoco tienen una importancia clara, siendo los jubilados los que tuvieron un impacto aumentando esta probabilidad en el primer periodo. Este efecto parece también alto en el segundo periodo pero no es estadísticamente significativo.
En cuanto a los grupos etarios, el grupo de entre 30 y 64 años impactó negativamente en la probabilidad de declarar desagrado por los precios, pero no fue significativo en el segundo periodo aunque sigue siendo negativo.

Como se observa de los que declaran desagrado por el precios es el de los brasileños y los argentinos. Aquí, hay una diferencia en el comportamiento, si bien los brasileños manifiestan desagrado por los precios, como vimos antes son ellos los que generan un alto gasto total, en cambio los argentinos manifiestan desagrado y coherentemente son los que menos gastan.

Una posible explicación tanto al bajo gasto de los argentinos y al alto de los brasileños así como al 


\begin{tabular}{|c|c|c|c|c|c|c|c|c|}
\hline \multirow[b]{2}{*}{ Var. Depend. } & \multicolumn{4}{|c|}{ Gasto Total } & \multicolumn{4}{|c|}{ Gasto en Compras (Shopping) } \\
\hline & Tobit $^{(\mathbf{b})}$ & Heckman $^{(\mathrm{c})}$ & Selección ${ }^{(\mathrm{c})}$ & $\mathrm{dP} / \mathrm{dx}$ & Tobit $^{(\mathbf{b})}$ & Heckman $^{(\mathrm{c})}$ & Selección ${ }^{(\mathrm{c})}$ & $d P / d x$ \\
\hline Brasil & $-0.01[-0,05]$ & $\begin{array}{r}0,16 \\
{[3,04]^{* * *}} \\
\end{array}$ & $-0.07[-0,92]$ & $-0,02$ & $0,52[1,26]$ & $0,17[2,17]^{* *}$ & $0,04[0,74]$ & 0,02 \\
\hline Argentina & $-1.05[-6,59]^{* * *}$ & $\begin{array}{r}-0,41[- \\
5,73] * * *\end{array}$ & $-0.40[-5,10]^{* * *}$ & $-0,10$ & $-1,38[-2,82]^{* * *}$ & $-0,23[-1,80]^{*}$ & $-0,24[-3,61] * * *$ & $-0,09$ \\
\hline EE.UU. & $-0,17[-0,92]$ & & & & $0,21[0,37]$ & & & \\
\hline G. Todo & $-0,09[-0,79]$ & $\begin{array}{r}0,13 \\
{[2,63]^{* * *}}\end{array}$ & $-0,13[-2,05]^{* *}$ & $-0,03$ & $0,43[1,21]$ & & & \\
\hline G. Gente & $0,11[0,99]$ & & & & $-0,33[-1,03]$ & & & \\
\hline G. Edificios & $0,28[2,19]^{* * *}$ & $-0,05[-0,9]$ & $0,25[3,04]^{* * *}$ & 0,05 & $0,51[1,32]$ & & & \\
\hline G. Playa & $-0,20[-1,60]$ & $0,09[1,58]$ & $-0,16[-2,22]^{* *}$ & $-0,04$ & $-1,56[-3,91]^{* * *}$ & $0,07[0,55]$ & $-0,29[-4,87]^{* * *}$ & $-0,10$ \\
\hline G. Higiene & $0,34[2,01]^{* *}$ & $0,12[1,69]^{*}$ & $0,15[1,48]$ & 0,03 & $-1,65[-2,94]^{* * *}$ & $0,17[1,01]$ & $-0,30[-3,47]^{* * *}$ & $-0,10$ \\
\hline G. Mer. Puerto & $0,51[2,55]^{* * *}$ & $0,12[1,41]$ & $0,37[2,60]^{*}$ & 0,07 & $-1,93[-3,03]^{* * *}$ & $0,08[0,45]$ & $-0,31[-3,13]^{* * *}$ & $-0,10$ \\
\hline D. Nada & $0,03[0,23]$ & & & & $0,23[0,52]$ & & & \\
\hline D. Precios & $-0,50[-2,47] * * *$ & $\begin{array}{r}-0,28[- \\
3,81]^{* * *} \\
\end{array}$ & $-0,17[-1,89]^{*}$ & $-0,04$ & $-0,89[-1,40]$ & & & \\
\hline D. Higiene & $0,09[0,30]$ & & & & $1,35[1,54]$ & & & \\
\hline D. Pobreza & $-0,48[-1,07]$ & & & & $-2,98[-2,00]^{* *}$ & $-0,42[-1,04]$ & $-0,45[-1,96]^{* *}$ & $-0,14$ \\
\hline 1ra Vez & $0,06[0,36]$ & & & & $-0,15[-0,30]$ & & & \\
\hline No. Visitas & $-0.00[-0,04]$ & & & & $-0,13[-0,81]$ & & & \\
\hline Profesional & $0,85[5,90]^{* * *}$ & $\begin{array}{r}0,35 \\
{[5,95]^{* * *}} \\
\end{array}$ & $0,36[5,18]^{* * *}$ & 0,08 & $0,63[1,44]$ & $0,25[3,30]^{* * *}$ & $0,02[0,44]$ & 0,01 \\
\hline Jubilado & $-0,02[-0,12]$ & & & & $0,23[0,41]$ & & & \\
\hline Patrón & $0,78[4,49]^{* * *}$ & $\begin{array}{r}0,47 \\
{[6,35] * * *}\end{array}$ & $0,25[2,88]^{* * *}$ & 0,05 & $0,58[1,11]$ & $0,46[4,63]^{* * *}$ & $-0,00[0,00]$ & 0,00 \\
\hline Empleado & $0,64[3,13]^{* * *}$ & $\begin{array}{r}0,43 \\
{[5,13]^{* * *}} \\
\end{array}$ & $0,17[1,64]^{*}$ & 0,04 & $1,44[2,38]^{* *}$ & $0,32[2,45]^{* * *}$ & $0,16[1,81]^{*}$ & 0,06 \\
\hline Hombre & $0,18[1,83]^{*}$ & $0,02[0,50]$ & $0,10[1,68]^{*}$ & 0,02 & $-0,25[-0,85]$ & & & \\
\hline ed36a64(a) & $0,44[2,48]^{* * *}$ & $0,13[1,61]$ & $0.19[1,96]^{* *}$ & 0,05 & $-0,24[-0,46]$ & & & \\
\hline ed 65 & $0,46[1,99]^{* *}$ & $\begin{array}{r}0,22 \\
{[2,57]^{* * *}} \\
\end{array}$ & $0,13[1,26]$ & 0,03 & $0,35[0,51]$ & & & \\
\hline Constante & $1,88[6,51]^{* * *}$ & $\begin{array}{r}3,29 \\
{[26,56]^{* * *}} \\
\end{array}$ & $0,82[7,26]^{* * *}$ & & $-4,05[-4,59] * * *$ & $3,96[8,15]^{* * *}$ & $-0,31[-6,45]^{* * *}$ & \\
\hline $\begin{array}{l}\text { Obs. no } \\
\text { censuradas }\end{array}$ & 2803 & 2803 & & & 1114 & 1114 & & \\
\hline Chi2 & (LR) $228.33^{* * *}$ & $\begin{array}{r}\text { (Wald) } \\
172.22^{* * *}\end{array}$ & & & (LR) $116.26^{* * *}$ & (Wald) $55.57 * * *$ & & \\
\hline media var. Ind. & 61.05 & 61.05 & No sesgo & $0,85^{(\mathrm{d})}$ & 24.65 & 24.65 & No sesgo & $0,33^{(\mathrm{d})}$ \\
\hline Predicción & 18.20 & 62.12 & & & 0.00 & 22.55 & & \\
\hline $\begin{array}{l}\text { Criterio Akaike } \\
\text { (AIC) }\end{array}$ & 14863.62 & 11134.33 & & & 9871.933 & 7431.546 & & \\
\hline
\end{tabular}

Tabla 8. Regresión de Tobit para el Gasto de los cruceristas en Uruguay 2009/2010*significación al 10\%,**significación al 5\%, *** significación al 1\%. (a) La encuesta de 2009/10 usa ed30-64. (b) Coeficientes y estadístico W entre paréntesis [] para el modelo Tobit. (c) Modelo de Heckman primera columna coeficientes, segunda columna ecuación selección y la tercera muestra el efecto en la probabilidad de gastar (d) probabilidad de gastar. Fuente: Cálculos propios en base a las encuestas de cruceros suministradas por el Ministerio de Turismo y Deporte de Uruguay

desagrado por los precios puede estar en la apreciación del tipo de cambio real. Los Índices de Tipo de Cambio Real Efectivo se encuentran definidos como la relación entre los índices de precios al consumo de nuestros principales socios comerciales con respecto a la economía doméstica, expresados en una misma moneda.

Como se puede apreciar en la figura 7 el tipo de cambio real con Argentina se ha apreciado continuamente desde 2000 acumulando una caída del $-54,2 \%$. Esto sin lugar a dudas hace que los argentinos tengan una percepción de Uruguay como un país caro en comparación a Argentina. Esto podría estar explicando la dependencia negativa de los argentinos con el gasto y la alta correlación positiva con el desagrado por los precios.

Por otro lado, nótese que el panorama es diferente con los brasileños. Con Brasil se produce una depreciación del tipo de cambio real del 36,8\%, es Brasil el que se ha encarecido con respecto al Uruguay desde el 2000. Si se recuerda que a pesar de existir una tendencia en ellos a declarar un desagrado por los precios igual realizan gastos, esto puede ser debido a que como se observa en el grafico existe una reciente tendencia de Uruguay a encarecerse respecto a Brasil desde el 2007. En la figura 7 se observa que después del máximo en 2007, el tipo de cambio real con Brasil se apreció, decreciendo un -11,02\% entre 2007 y 2010.

\section{Conclusiones}

El mercado de cruceros ha presentado un gran crecimiento en Uruguay en los últimos años. La diversificación en este 


\begin{tabular}{|c|c|c|c|c|}
\hline \multirow[b]{2}{*}{ Var. Depend. } & \multicolumn{4}{|c|}{ Gasto en Alimentación } \\
\hline & Tobit $^{(\mathbf{b})}$ & Heckman $^{(c)}$ & Selección ${ }^{(c)}$ & dP/dx \\
\hline Brasil & $-0.51[-1,06]$ & & & \\
\hline Argentina & $-2.05[-3,54]^{* * *}$ & $-0,37[-3,26]^{* * *}$ & $-0,18[-2,79]^{* * *}$ & -0.04 \\
\hline EE.UU. & $0,62[0,92]$ & & & \\
\hline G. Todo & $-2,92[-6,65]^{* * *}$ & $0,60[5,08]^{* * *}$ & $-0,50[-7,78]^{* * *}$ & -0.12 \\
\hline G. Gente & $-2,06[-5,18]^{* * *}$ & $0,35[3,28]^{* * *}$ & $-0,31[-5,28]^{* * *}$ & -0.08 \\
\hline G. Edificios & $-1,21[-2,58]^{* * *}$ & $0,14[1,12]$ & $-0,19[-2,65]^{* * *}$ & -0.05 \\
\hline G. Playa & $-0,60[-1,30]$ & $0,05[0,46]$ & $-0,14[-2,08]^{* *}$ & -0.04 \\
\hline G. Higiene & $-2,16[-3,19]^{* * *}$ & $0,46[2,46]^{* * *}$ & $-0,33[-3,32]^{* * *}$ & -0.08 \\
\hline G. Mer. Puerto & $0,61[0,89]$ & $0,27[1,66]^{*}$ & $0,06[0,56]$ & 0.02 \\
\hline D. Nada & $-0,74[-1,48]$ & & & \\
\hline D. Precios & $0,56[0,79]$ & & & \\
\hline D. Higiene & $1,48[1,50]$ & $-0,12[-0,55]$ & $0,34[2,47]^{* * *}$ & 0.10 \\
\hline D. Pobreza & $-4,02[-2,08] * *$ & $0,55[1,06]$ & $-0,54[-1,88]^{*}$ & -0.11 \\
\hline 1ra Vez & $0,34[0,58]$ & & & \\
\hline No. Visitas & $0,21[1,17]$ & & & \\
\hline Profesional & $-1,25[-2,49] * * *$ & $0,58[4,84]^{* * *}$ & $-0,26[-3,59]^{* * *}$ & -0.07 \\
\hline Jubilado & $-3,07[-4,53]^{* * *}$ & $0,71[4,82]^{* * *}$ & $-0,51[-6,20]^{* * *}$ & -0.12 \\
\hline Patrón & $-1,31[-2,13]^{* *}$ & $0,78[5,22]^{* * *}$ & $-0,30[-3,40]^{* * *}$ & -0.07 \\
\hline Empleado & $0,45[0,65]$ & $0,55[3,28]^{* * *}$ & $0,00[0,03]$ & 0.00 \\
\hline Hombre & $1,35[3,83]^{*}$ & $-0,11[-1,24]$ & $0,20[3,80]^{* * *}$ & 0.05 \\
\hline ed36a64 (a) & $-1,10[-1,84]^{*}$ & $0,20[1,89]^{* *}$ & $-0,11[-1,77]^{*}$ & -0.03 \\
\hline ed65 & $-0,60[-0,74]$ & & & \\
\hline Constante & $-3,19[-3,18]^{* * *}$ & $3,81[21,29] * * *$ & $-0,22[-2,59]^{* * *}$ & \\
\hline $\begin{array}{l}\text { Obs. no } \\
\text { censuradas }\end{array}$ & 661 & 661 & & \\
\hline Chi2 & (LR) $205.15^{* * *}$ & (Wald) $91.68^{* * *}$ & & \\
\hline media var. Ind. & 5.70 & 5.70 & sesgo & $18 \%$ (d) \\
\hline Predicción & 0.00 & 1.29 & & \\
\hline $\begin{array}{l}\text { Criterio Akaike } \\
\text { (AIC) }\end{array}$ & 6415.493 & 4932.367 & & \\
\hline
\end{tabular}

Tabla 9. Regresión de Tobit para el Gasto de los cruceristas en Uruguay 2009/2010. *significación al 10\%, **significación al 5\%, *** significación al 1\%. (a) La encuesta de 2009/10 usa ed30-64. (b) Coeficientes y estadístico $\mathrm{W}$ entre paréntesis [] para el modelo Tobit. (c) Modelo de Heckman primera columna coeficientes, segunda columna ecuación selección y la tercera muestra el efecto en la probabilidad de gastar.(d) probabilidad de gastar. Fuente: Cálculos propios en base a las encuestas de cruceros suministradas por el Ministerio de Turismo y Deporte de Uruguay

\begin{tabular}{|l|r|r|r|r|}
\hline \multirow{2}{*}{ Rubro } & \multicolumn{2}{|c|}{$\mathbf{2 0 0 8 / 0 9}$} & \multicolumn{2}{c|}{$\mathbf{2 0 0 9 / 1 0}$} \\
\cline { 2 - 5 } & $\begin{array}{c}\text { Gasto } \\
\text { Promedio }\end{array}$ & $\begin{array}{c}\text { Probabilidad } \\
\text { media }\end{array}$ & $\begin{array}{c}\text { Gasto } \\
\text { Promedio }\end{array}$ & $\begin{array}{c}\text { Probabilidad } \\
\text { media }\end{array}$ \\
\hline Total & 62,54 & $86 \%$ & 62,12 & $85 \%$ \\
\hline Compras & 22,16 & $30 \%$ & 22,55 & $33 \%$ \\
\hline Alimentos & $6,13 *$ & $21 \%$ & $5,70 *$ & $18 \%$ \\
\hline * Es la media de la variable \\
\hline
\end{tabular}

Tabla 10. Probabilidades medias y Gasto medio de generar los distintos tipos de gasto. Fuente: Cálculos propio en base a las encuestas 2008/09, 2009/10 del Ministerio de Turismo y Deporte. tipo de turismo y en los paquetes que se ofrecen ha permitido el acceso a los cruceros a una gama diversificada de turistas.

El arribo de cruceros y el desembarco de cruceristas a Uruguay han crecido desde la temporada en 2004/05, con un marcado crecimiento en Punta del Este que ha superado a Montevideo e incluso a otros destinos internacionales como Buenos Aires. De acuerdo a los arribos de 2009/10 la mayoría de estos turistas son mujeres $(59,03 \%)$, en cuanto a la edad la mayoría se encuentra entre los 30 y 64 años $(61,12 \%)$. El 52,8\% de estos visitantes proviene de Brasil, mientras que los restantes en importancia son argentinos

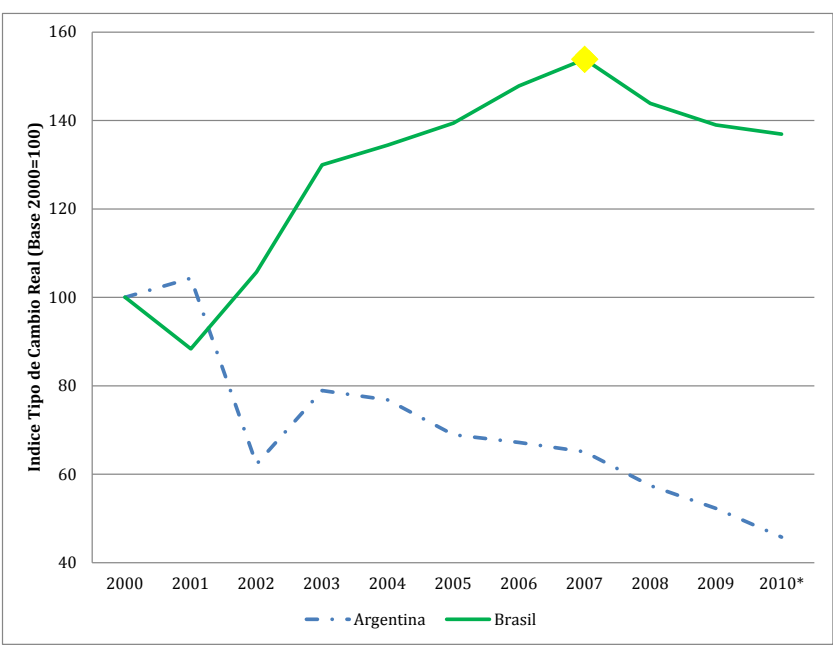

Figura 7. Evolución del Tipo de cambio real con Argentina y con Brasil periodo 2000-2010* Promedio hasta noviembre 2010. Fuente: Cálculos en base a los índices proporcionados por el $\mathrm{BCU}$.

$(23,4 \%)$ y norteamericanos (12,2\%). En 2009/10 arribaron 292.048 cruceristas que gastaron en promedio U\$S 61,05 por persona. Los Uruguayos y los Argentinos son los turistas que gastan menos por persona con U\$S 8,67 y U\$S 35,78. Sin embargo, los brasileños y el resto de los del mundo (excluido EEUU, Europa y América) tienen el gasto por persona más alto de U\$S 71,15 y U\$S 90,71 por persona.

En este contexto, el presente trabajo analizó los determinantes del gasto de los cruceristas de acuerdo a las encuestas de 2008/09 y 2009/10. En cuanto al gasto total, si bien el turistas brasileño determina un incremento en la probabilidad de gastar de 5 puntos porcentuales en el primer periodo (nótese que la probabilidad de gastar es $86 \%$ ), el mismo tipo de visitante reduce la probabilidad de gastar en 2 puntos en 2009/10. Sin embargo, para ambos periodos el crucerista brasileño aumenta el gasto en $21 \%$ y un $16 \%$ en el segundo. Esto puede ir de acuerdo con la hipótesis de que Uruguay se ha hecho más barato que Brasil desde el 2000, pero que desde el 2007 se ve un 
pequeño encarecimiento de Uruguay, respecto a Brasil. El turista argentino impacta negativamente tanto en el nivel de gasto como en la probabilidad de realizar el gasto. Este visitante reduce el gasto en $-29 \%$ y $-41 \%$ y la probabilidad de gasto en 15 y 10 puntos porcentuales. El desagrado por los precios reduce la probabilidad de gasto en 7 y 4 puntos porcentuales, sin embargo una vez que se decide realizar el gasto, el desagrado por el precio impacta positivamente en el gasto en el primer periodo y negativamente en el segundo, esto también puede estar relacionado con una explicación ligada al gasto de los brasileños. Por último, se puede definir el perfil del crucerista que ha impactado con un mayor gasto es el brasileño patrón, comerciante, industrial, productor agropecuario, empresario con más de 65 años. Mientras que el perfil más negativo es el de un visitante argentino menor de 65 años que ha manifestado desagrado por los precios.
En cuanto al gasto en compras se observa que en el primer periodo es significativa la reducción del gasto por parte de los argentinos en $43 \%$ y el aumento del gasto por parte de los brasileños en $15 \%$, en el segundo periodo se mantienen los signos, pero lo que es significativo es la decisión de comprar (la cual tiene una probabilidad de $30 \%$ y de $32 \%$ en cada periodo), la cual aumenta en el caso de los brasileños en 2 puntos porcentuales y disminuye en 9 puntos en el caso de los argentinos. Por otro lado, la variable desagrado por la pobreza también ha reducido significativamente la probabilidad de comprar en 8 y 14 puntos porcentuales. En cuanto al gasto en alimentación la variable más importante es la de género en donde los hombres son los que tienen un impacto positivo significativo solo en la probabilidad de gasto en alimentación con 9 y 5 puntos porcentuales.

Si bien se realizó un estudio sobre la demanda, se debe

\begin{tabular}{|c|c|c|c|c|c|c|c|c|c|c|c|c|}
\hline \multirow[b]{2}{*}{ Precios } & \multicolumn{6}{|c|}{ año 2008/2009 } & \multicolumn{6}{|c|}{ año 2009/2010 } \\
\hline & Coef. & $\mathrm{dF} / \mathrm{dx}$ & Wald & Coef. & $\mathbf{d F} / \mathbf{d x}$ & Wald & Coef. & $\mathrm{dF} / \mathrm{dx}$ & Wald & Coef. & $\mathrm{dF} / \mathrm{dx}$ & Wald \\
\hline Brasil & 1.1599 & 0.097 & {$[6.54]^{* *}$} & 0.8264 & 0.131 & {$[7.16]^{* *}$} & 0.4255 & 0.005 & {$[2,72]^{* *}$} & 0.4806 & 0.006 & {$[3,60]^{* *}$} \\
\hline Argentina & 0.8378 & 0.115 & {$[4.15]^{* *}$} & 0.8086 & 0.152 & {$[5.92]^{* *}$} & 0.8354 & 0.017 & {$[4,89]^{* *}$} & 0.9222 & 0.023 & {$[6,30]^{* *}$} \\
\hline EE.UU. & -0.3256 & -0.04 & {$[-1.11]$} & & & & -0.4339 & -0.003 & {$[-1,57]$} & & & \\
\hline G. Todo & -0.1128 & -0.052 & {$[-0.56]$} & & & & 0.6462 & 0.010 & {$[5,38]^{* *}$} & 0.5814 & 0.009 & {$[5,08]^{* *}$} \\
\hline G. Gente & 0.0738 & 0.022 & {$[0.40]$} & & & & 0.1435 & 0.002 & {$[1,39]$} & & & \\
\hline G. Playa & 0.0393 & -0.006 & [0.15] & & & & 0.4941 & 0.008 & {$[4,20]^{* *}$} & 0.4690 & 0.008 & {$[4,14]^{* *}$} \\
\hline G. Edificios & -0.4389 & -0.04 & {$[-1.57]$} & & & & 0.1880 & 0.002 & {$[1,42]$} & & & \\
\hline G. Higiene & -0.2354 & -0.026 & {$[-0.63]$} & & & & 0.5860 & 0.013 & {$[3,86]^{* *}$} & 0.5569 & 0.013 & {$[3,76]^{* *}$} \\
\hline $\begin{array}{l}\text { G. Mer. } \\
\text { Puerto } \\
\end{array}$ & -0.5879 & -0.042 & {$[-1.18]$} & & & & 0.1235 & 0.002 & {$[0,53]$} & & & \\
\hline D. Nada & & & & & & & -3.2187 & -0.390 & {$[-15,89]^{* *}$} & -3.1827 & -0.395 & {$[-15,33]^{* *}$} \\
\hline D. Higiene & & & & & & & -1.3513 & -0.004 & {$[-4,84]^{* *}$} & -1.2787 & -0.004 & {$[-4,48]^{* *}$} \\
\hline D. Pobreza & & & & & & & -1.4464 & -0.004 & {$[-3,07]^{* *}$} & -1.3293 & -0.004 & {$[-2,65]^{* *}$} \\
\hline 1 ra Vez & 0.6165 & 0.043 & {$[2.35]^{* *}$} & 0.3807 & 0.044 & {$[2.19]^{* *}$} & -0.0428 & 0.000 & {$[-0,26]$} & & & \\
\hline No. Visitas & 0.1783 & 0.015 & {$[2.38]^{* *}$} & 0.1195 & 0.015 & {$[2.40]^{* *}$} & 0.0306 & 0.000 & {$[0,65]$} & & & \\
\hline Gasto Total & -0.0001 & 0 & {$[-0.16]$} & & & & 0.0000 & 0.000 & {$[0,04]$} & & & \\
\hline Profesional & 0.1925 & 0.003 & [0.93] & & & & -0.0266 & 0.002 & {$[-0,18]$} & & & \\
\hline Jubilado & 0.7146 & 0.035 & {$[3.04]^{* *}$} & 0.1815 & 0.025 & {$[1.68]^{*}$} & 0.1423 & 0.002 & {$[0,79]$} & & & \\
\hline Patron & 0.3572 & 0.014 & {$[1.40]$} & & & & 0.0276 & 0.002 & {$[0,16]$} & & & \\
\hline Empleado & 0.5999 & 0.01 & {$[1.85]^{*}$} & 0.0416 & 0.005 & {$[0.24]$} & -0.0831 & -0.002 & {$[-0,36]$} & & & \\
\hline Hombre & -0.1704 & -0.015 & {$[-1.19]$} & & & & 0.0188 & 0.001 & {$[0,19]$} & & & \\
\hline ed30a64(a) & -0.3703 & -0.015 & {$[-1.72]^{*}$} & -0.0979 & -0.013 & {$[-1.01]$} & -0.1327 & -0.003 & {$[-0,68]$} & & & \\
\hline ed65 & -0.2025 & -0.004 & -0.71 & & & & -0.3221 & -0.002 & {$[-1,36]$} & & & \\
\hline Constante & -1.5892 & 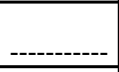 & $\begin{array}{r}{[-} \\
4.04]^{* *} \\
\end{array}$ & -2.2876 & 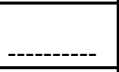 & $\begin{array}{r}{[-} \\
10.84]^{* *} \\
\end{array}$ & -0.9385 & --.----.- & {$[-3,28]^{* *}$} & -1.0366 & & {$[-8,57]^{* *}$} \\
\hline $\begin{array}{l}\text { Observacione } \\
\mathrm{s}\end{array}$ & 447 & & & 1803 & & & 3347 & & & 3347 & & \\
\hline Obs. P & $33 \% / 30 \%$ & & & $8 \% / 7 \%$ & & & $9 \% / 0,3 \%$ & & & $9 \% / 0,4 \%$ & & \\
\hline Wald Chi2 & $85.33 * *$ & & & $67.60 * *$ & & & $360.77 * *$ & & & $287.27 * *$ & & \\
\hline AIC & 513 & & & 971 & & & 965 & & & 948 & & \\
\hline
\end{tabular}

Tabla 11. Modelo Probit probabilidad del desagrado por los precios de los cruceristas en Uruguay, periodo 2008 a 2010*significación al 10\%, **significación al 5\%. (a) La encuesta de 2008/09 usa ed36-64. Fuente: Cálculos propios en base a las encuestas de cruceros suministradas por el Ministerio de Turismo y Deporte de Uruguay 
señalar que aún hay cosas por hacer del lado de la oferta. Muchos de los cruceristas manifiestan que a pesar de que desean realizar compras muchas veces encuentran pocos lugares abiertos. Esto ocurre especialmente los fines de semana, en donde los negocios cercanos a los puertos en su mayoría permanecen cerrados, desaprovechando la oportunidad de colocar sus productos.

Se estudió de qué depende que alguien declare desagrado por los precios ya que podría ser un indicador de reducción en el gasto, ya que esta variable siempre mantuvo una dependencia negativa con el gasto. En ambos casos el impacto marginal es más alto en el caso de los argentinos que el de los brasileños. Un argentino aumenta la probabilidad en $15,2 \%$ y $1,4 \%$ en cada periodo y un brasileño en $13,1 \%$ y $0,4 \%$. Por otro lado, los norteamericanos parecen no disgustarse por los precios siendo esto significativo en el segundo periodo con una reducción en $0,3 \%$.

Se puede observar una diferencia en el comportamiento, si bien los brasileños manifiestan desagrado por los precios, son ellos los que generan un alto gasto total, en cambio los argentinos manifiestan desagrado pero son quienes menos gastan. Una posible explicación a esto es que el tipo de cambio real con Argentina se ha apreciado continuamente desde 2000 acumulando una caída del -54,2\%. Esto sugeriría que los argentinos pueden tener una percepción de Uruguay como un país caro en comparación a Argentina. Por el contrario, con Brasil se produce una depreciación del tipo de cambio real del $36,8 \%$, es Brasil el que se ha encarecido con respecto al Uruguay desde el 2000. Solamente a partir del 2007 aparece una pequeña reversión y Uruguay se comienza a encarecer un poco más respecto a Brasil, aquí puede estar la explicación de porqué el turista brasileño declare desagrado por los precios a partir de 2008/09 pero igual genere un alto gasto.

Hubiera sido interesante además estudiar de qué depende la probabilidad de regresar a Uruguay en el futuro. Esto podría indicar el perfil de las personas propensas a retornar al país, así como aquellos factores que encontraron en el país y jugaron a favor o en contra. Para esto se necesitaría incluir la pregunta de si piensa regresar al país.

Es de señalar que también sería importante estudiar la influencia sobre el gasto de variables como la cantidad de horas que el turista pasa fuera del crucero, el nivel de ingreso de los visitantes, el nivel educativo y el estado civil que no se pudieron analizar por falta de datos. Estas variables han demostrado tener importancia en otros casos.

(Lynch, 2004) analizando a los cruceristas en Bar Harbor sugiere que quien pasa más tiempo en el puerto tiene más tiempo para realizar grandes compras. En el caso de Bar Harbor los cruceros estaban 9 horas, pero los pasajeros estaban 5 horas y 20 minutos en el puerto. Si se tuviera información sobre las horas se podría analizar el caso en
Uruguay. Se podría ofrecer incentivo a las líneas de cruceros para que lleguen temprano en la mañana a los puertos y salgan tarde en la noche, dejando que los visitantes tengan más tiempo para gastar. Una forma para que los cruceros permanezcan más tiempo podría ser con descuentos en los impuestos portuarios a aquellos cruceros que permanezcan por más de 10 o 12 horas. Esto podría permitir que los turistas tuvieran más tiempo para evaluar un posible retorno al país.

Es de destacar también que si bien el número de cruceros y el gasto de los cruceristas ha crecido en todo el periodo, se ha notado que esta tasa se ha desacelerado. Esto ha sucedido con más fuerza en Montevideo, en donde la tasa anual de crecimiento de cruceros desembarcados pasa de $60,82 \%$ en $2005 / 06$ a un $0,57 \%$ en $2009 / 10$. Un estudio más profundo implicaría analizar la posibilidad de un congestionamiento de cruceros respecto a las facilidades portuarias, tal vez el estudio de la posibilidad de ampliación del puerto o de puertos adicionales en el país.

\section{Bibliografía}

Aguiló, E., \& Juaneda, N.

2000 "Tourist expenditure for mass tourism markets", Annals of Tourism Research, vol. 27(3), pp. 624-637.

Besteiro Rodriguez, B.

2004 "El desarrollo del turismo náutico en Galicia", Cuadernos de Turismo, no. 13, pp. 145-163.

Brida, J., Risso, W.

2010 "Cruise Passengers Expenditure analysis and probability of repeat visits to Costa Rica: A Cross-Section data analysis", Tourism Analysis, Vol. 15 (4)

Brida, J., Bukstein,D., Garrido,N., Tealde, E., zapata, S.

2010 Impacto económico del turismo de cruceros. Un análisis del gasto de los cruceristas que visitan el Caribe colombiano, documento de investigación, Universidad ORT, Uruguay.

Cameron, A., Trivedi, P.

2009 Microeconometrics Using STATA, Stata Press, Texas, US

Chase, G., Alon, I.

2002 "Evaluating the economic impact of cruise tourism: a case study of Barbados", Anatolia: an international journal of tourism and hospitality research, vol. 13(1)

Cuellar-Río, M., Kido-Cruz, M.

2008 "Perfil y análisis del gasto del crucerista: El caso de Bahías de Huatulco (México)", Cuadernos de Turismo, vol. 22 , pp. 47-78

Dwyer, L., Forsyth, P.

1998 "Economic significance of cruise tourism", Annals of Tourism Research, vol. 25, no. 2, pp. 393-415.

Esteban Chapapría, V.

2000 "El planeamiento de infraestructuras para el turismo Náutico", Cuadernos de Turismo, no 6, pp. 2944 . 
Ferradás Carrasco, S.

2001 "La relevancia del turismo náutico en la oferta turística", Cuadernos de Turismo, no 7, pp. 67-80.

Ferradás Carrasco, S

2002 "El turismo náutico en el Mediterráneo", Cuadernos de Turismo, no 9, pp. 19-32.

\section{FCCA}

2010 Cruise Industry Overview - 2010: State of the cruise industry, Florida-Caribbean Cruise Association, http://www.f-cca.com/ downloads/2010-overview-book_Cruise-Industry-Overview-and-Statistics.pdf.

Lynch, C.

2004 Cruise tourism in Bar Harbor, Maine: An analysis of likelihood of passenger return and passenger expenditures in port, Thesis submitted to Graduate School, The University of Maine, United States, http://www.library.umaine. edu/theses/pdf/LynchCP2004.pdf

Long, J. Scott

1997 Regression Models for Categorical and Limited Dependent Variables. Thousand Oaks, CA: Sage Publications

Losano,P., Torrejon, C.

2006 "El turismo de cruceros", Boletín Electrónico Intercambios, año 5, no. 26 http://fatu.uncoma. edu.ar/boletin/2006/mar_06.html\#losano

McCarthy, J.

2003 "The cruise industry and port city regeneration: the case of Valleta", European Planning Studies, vol. 11, no. 3, pp. 341-350.

Mescon, T.S., Vozikis, G.

1985 "The economic impact of tourism at the port of Miami", Annals of Tourism Research, vol. 12 , pp. 515-528.

Ministerio de Turismo

2010 Anuario Estadístico 2010, Ministerio de Turismo y Deportes, Montevideo, Uruguay, http:// www.mintur.gub.uy/images/stories/pdf/estadisticas/anuarios/Anuario2010.pdf

Seidl, A., Guiliano, F., Pratt, L.

2006 "Cruise tourism and community economic development in Central America and the Caribbean: the case of Costa Rica", Pasos. Revista de Turismo y Patrimonio Cultural, vol. 4, no 2, pp. 213-224.

Seidl, A., Guiliano, F., Pratt, L.

2007 "Cruising for colones: cruise tourism economics in Costa Rica", Tourism Economics, vol. 13, no 1 , pp. 67-85.

Song, H., \& Li, G.

2008 "Tourism demand modeling and forecastingA review of recent research", Tourism Management, vol. 29(2), pp. 203-220.

Tobin, J.

1958 "Estimation of relationships for limited depen- dent variables". Econometrica 26, 24-36.

Wood, R.

2000 "Caribbean cruise tourism: globalization at sea", Annals of Tourism Research, vol. 27, no 2, pp. 345-370.
Recibido:

$25 / 02 / 2011$

Reenviado:

$05 / 09 / 2011$

Aceptado:

09/09/2011

Sometido a evaluación por pares anónimos 